\title{
Use of wearable inertial sensors for the assessment of spatiotemporal gait variables in children: A systematic review
}

\author{
Paulo Roberto Fonseca Junior ${ }^{1}$ (D) , Renata Calhes Franco de Moura $^{2}$ (i) , Cláudia Santos Oliveira ${ }^{3}$, Fabiano Politti ${ }^{1}$ (i) \\ ${ }^{1}$ Universidade Nove de Julho, Programa de Pós-graduação em Ciências da Reabilitação, São Pau- \\ lo, SP, Brasil; ' ${ }^{2}$ Universidade de Mogi das Cruzes, Departamento de fisioterapia, Mogi das Cru- \\ zes, SP, Brasil; ${ }^{3}$ Centro Universitário UniEVANGELICA, Anápolis, GO, Brasil.
}

\begin{abstract}
Aim: The present study aimed to perform a literature review on the use of wearable inertial sensors for gait analysis of children in clinical practice. Methods: Searches were performed in the MEDLINE, EMBASE, Cochrane Library, and PEDro databases for studies involving children or adolescents submitted to gait analysis with the use of wearable inertial sensors. No restrictions were imposed regarding the date of publication or language. Results: Three hundred twenty articles were retrieved, 14 of which met the eligibility criteria and were selected for the present systematic review. Two independent reviewers assessed the risk of bias and study quality using the ROBINS-I and AXIS scale. The studies included in the present review reported multiple outcomes of kinematic gait assessments calculated from the signals provided by the wearable sensors, performed in a hospital setting, outpatient clinic, and a familiar environment, with several types of pediatric conditions. Conclusion: The findings suggest that wearable sensors are effective for the evaluation of quantitative gait variables in children with different pediatric conditions, enabling an objective analysis that should prove useful in the processes of clinical diagnosis and rehabilitation. However, given the relatively small number of studies published on this topic, it is difficult to make strong recommendations regarding the most appropriate equipment, sensor placement, and outcomes for assessing gait in children.
\end{abstract}

Keywords: gait analysis; wearable inertial sensors; pediatrics; clinical application.

\section{Introduction}

Gait analysis is the systematic quantitative measurement, description, and assessment of human locomotion and therefore plays an important role in clinical practice ${ }^{1}$. This type of analysis provides objective information on a patient's functional level and can be used to evaluate the effectiveness of rehabilitation programs as well as the success of surgical procedures ${ }^{2}$.

The kinematics of gait in children is similar to that of adults. However, discrepant results have been reported with regard to the underlying kinetics, providing support for the hypothesis that children lack the neuromuscular maturity for the production of an adult-like gait pattern ${ }^{3}$. Gold standard gait analysis methods successfully developed and applied in several gait laboratories involve a multi-camera motion capture system and force plate with the capability of measuring ground-reaction forces ${ }^{4-6}$. While these gait analysis methods provide detailed information on kinematic and kinetic variables, the data acquisition systems are limited to laboratory use and require expensive equipment with lengthy setup and post-processing times s $^{7,8}$.

An alternative gait analysis method involving the use of wearable inertial sensors has shown great prospects in the last two decades ${ }^{9,10}$. Sensors and recording equipment are relatively compact, portable, and less expensive compared with traditional laboratory-based and can be used to collect data on human movements, such as spatiotemporal gait variables, in environments and contexts where the use of traditional equipment is not possible. This method has been validated for gait analysis in children with typical development and those with cerebral palsy as well as healthy subjects, elderly subjects, and patients with Parkinson's disease ${ }^{11-15}$. In the evaluation of spatiotemporal gait variables, motion sensors are worn or attached to various parts of the volunteer's body, such as the lower back, ankles, foot, and waist ${ }^{9}, 16$. There are different types of motion sensors and systems, such as accelerometers, gyroscopes, and magnetoresistive sensors. A single type or combined sensor system with multiple types of sensors can be used for the analysis of the gait. An accelerometer is a type of inertial sensor that measures acceleration along its sensitive axis. Acceleration is measured electrically using physical changes in the displacement of the proof mass attached to a mechanical suspension system 
in relation to a reference frame ${ }^{9}$.

A gyroscope is an angular velocity sensor and is based on the measurement of force proportional to the angular speed of rotation in a rotating frame. For example, a gyroscope attached to the feet or legs enables the determination of angular velocities and angles during gait, which can assist in the reorganization of the various gait phases, which is usually combined with an accelerometer to achieve a more complete initial sensing system. Magnetoresistive sensors are based on the magnetoresistive effect that can estimate changes in the orientation of a body segment in relation to the magnetic North or the vertical axis. Such sensors provide information that cannot be determined by accelerometers or the integration of gyroscope signals ${ }^{9}$.

The present systematic review aimed to summarize studies in which gait analysis was performed with the aid of inertial sensors to determine whether these tools use practicality, a variety of information about different temporal spaces in children during locomotion and shows the types of conditions that are analyzed using inertial sensors in clinical practice.

\section{Methods}

\section{Protocol and registration}

This review was conducted in accordance with the Preferred Reporting Items for Systematic Reviews and Meta-Analyses (PRISMA statement) and is registered in the PROSPERO database (CRD42017079882).

\section{Data sources and search strategy}

The MEDLINE (PubMed), EMBASE, Cochrane Library, and PEDro databases were searched for relevant articles using the Medical Subject Headings (MeSH) of the U.S. National Library of Medicine. The following terms were used for the literature search: 'infant', 'child', 'children', 'adolescent', 'walk', 'walking', 'locomotor', 'gait', 'sensor', 'gyroscope', 'inertial', 'acceleration' and 'accelerometer'. Specifically, for inclusion in the present review, papers were required to have the terms ('infant' OR 'child' OR 'children') AND ('walk' OR 'walking' OR 'locomotion' OR 'gait') AND ('sensor' OR 'gyroscope' OR 'inertial' OR 'accelerometer' OR 'acceleration') in the title and/or abstract [appendix]. In addition to the systematic electronic database search, a targeted search of the bibliographies of relevant articles was also performed to identify any further studies for inclusion. In addition, the reference lists of included studies were manually searched to identify further potentially relevant published papers. The authors did not obtain any kind of support or funding to perform this process of searching and extracting data.

\section{Study selection}

Two independent researchers analyzed the title and abstract of the articles retrieved during the search of the databases. When insufficient information was found in the title and abstract to make a decision regarding eligibility, the full text was read. Pre-selected articles were submitted to full-text analysis to determine inclusion in the review based on the eligibility criteria. In cases of divergence of opinion or doubts regarding the relevance of the article, a third researcher analyzed the text in question to reach a consensus.

\section{Eligibility criteria}

The following inclusion criteria were randomized controlled clinical trial or cross-sectional study (no restrictions were imposed regarding the date of publication or language), involving children $(<=12$ years of age) submitted to gait analysis using wearable inertial sensors. The exclusion criteria were case study, cohort study, review study, pilot study, protocol study, individuals $>12$ years of age.

\section{Quality assessment}

The pre-selected non-randomized trials were submitted to an appraisal of methodological quality using the Risk of Bias In Non-Randomized Studies of Interventions (ROBINS-I) ${ }^{18}$ to investigate the robustness of the results regarding each of the 'risks of bias' components. For cross-sectional studies, the Appraisal Tool for Cross-Sectional Studies (AXIS scale) was used ${ }^{19}$. The classification of the studies was performed by two independent researchers (R.C.F.M. and C.S.O.) blinded to the objectives of the present review. In cases of a divergence of opinion, a third researcher made the decision regarding the score.

\section{Results}

Three hundred twenty articles were retrieved from the databases and other sources searched. After the analysis of the titles, abstract and complete texts, and the quality appraisal using the ROBINS-I and AXIS scale (Table 1), only fourteen articles met the eligibility criteria. Figure 1 displays a flowchart of the selection process.

\section{Study design and methodological quality}

The studies included in this review used different types of portable inertial sensors to observe or identify differences in gait variables among healthy individuals and/or those with a disease during locomotion activities.

Three nonrandomized studies ${ }^{21-23}$ were found. After assessment of methodological quality by the ROBINS-I 
scale, showing strengths in the bias categories after the start of interventions (last four categories), such as deviations from intended interventions, missing data, measurement of outcomes, and selective reporting of results. However, the three studies failed to adequately address baseline confounding before the interventions began, presenting biases such as the use of a convenience sample with different group sizes $^{21}$, a lack of similarity regarding baseline prognostic factors and type of intervention ${ }^{22}$, and time-varying confounding due to the switch between interventions compared between individuals ${ }^{23}$.

Twelve cross-sectional studies were included in this review ${ }^{11,13,17,20,24-30}$. The reliability of these studies was considered good on nearly all items of the AXIS scale, with suitable systematic interpretations and appropriate evaluations. Sample size justification is crucial, as sample size profoundly affects the significance of the outcomes of a study. Three cross-sectional studies ${ }^{11,27,26}$ either did not present a detailed description of the population or had a small sample without describing the methods used to determine the sample size. Therefore, the conclusions drawn from these studies may be inaccurate.

\section{Types of pediatric conditions analyzed with wearable inertial sensors}

Gait analysis with the use of wearable inertial sensors was performed in a hospital setting ${ }^{26,30}$, outpatient clinic $^{11,13,17,21,23-25,28,29}$ and a familiar environment ${ }^{20,27}$, with healthy children ${ }^{11}$ divided by age group ${ }^{17}$ and $\operatorname{sex}^{20}$, children with prelingual deafness ${ }^{22}$, Duchenne muscular dystrophy ${ }^{24}$, idiopathic toe walking ${ }^{27,28}$, spina bifida ${ }^{13}$ and cerebral palsy, including those with unilateral or bilateral spasticity ${ }^{23,25,29}$, hemiplegia ${ }^{30}$, diplegia, dystonia ${ }^{26}$, and ataxia ${ }^{21}$.

Besides movement analysis of the lower limbs during gait, some studies also assessed the use of wearable inertial sensors to distinguish patients with different levels of functional status ${ }^{17,21,22}$ and stages of disease progression ${ }^{24}$ or to compare different therapeutic gait interventions ${ }^{26,23}$.

\section{Sensor type and placement}

Several types of inertial sensors were used to evaluate the quantitative gait variables in children. Seven studies included in this review used complete inertial measurement units consisting of accelerometers, gyroscopes, and magnetometers ${ }^{11,13,17,20,23,25,29}$. Three studies used accelerometers and gyroscopes ${ }^{22,24,26}$. Three studies only used an accelerometer ${ }^{21,27,28}$ and one study only used a gyroscope ${ }^{30}$.

Similarly, different protocols were described regarding the placement of the wearable sensors. Among the 15 studies included, nine used more than one inertial sensor on the body $11,13,17,20-22,25,26,30$. Eleven studies reported placing a wearable sensor in either the lumbar or sacral region ${ }^{11,13,17,23-27,29}$. Four studies placed wearable sensors on the feet ${ }^{11,22,28,30}$.
Three studies placed wearable sensors on the legs $s^{21,26,30}$. Two studies placed wearable sensors on the ankles ${ }^{17,13}$ and three studies placed wearable sensors on the shins ${ }^{11,24,30}$. Some studies placed an inertial sensor in locations far from the lower limbs, such as the head, sternum, chest, and wrist $^{13,17,20,21,25}$. However, the aims of these studies included an evaluation of upper body acceleration in the participants. Table 2 displays details on the studies included in this review, including the specific type and placement of the sensors.

\section{Gait assessment procedures}

Three studies used wearable sensors to assess walking during clinical tests, such as the TGMD-2 locomotion subtest, which consists of six tasks (run, gallop, hop, leap, horizontal jump and slide $)^{17}, 10$-meter walk test ${ }^{13,24}$, Timed Up and Go test, Obstacles test and Curb test ${ }^{13}$. Other studies used the wearable sensors during assessments of straightline walking at a self-selected pace $^{31}$ with distances of five $^{23,29}, \operatorname{six}^{21}$, seven ${ }^{11}, \operatorname{ten}^{22,25}$, and fifteen meter ${ }^{27}$. In the study by Chen et $\mathrm{al}^{26}$, the distance was delineated by an exact number of consecutive steps to be performed ( 30 steps). Pendharkar et $\mathrm{a}^{28}$ evaluated each child walking on a treadmill for two minutes.

Gait variables obtained with wearable inertial sensors

The studies included in the present review reported multiple outcomes of kinematic gait assessments calculated from the signals provided by the wearable sensors. The most commonly-reported quantitative variables were anteroposterior (AP) acceleration ${ }^{13,17,20,25-26,28,30}$, mediolateral (ML) acceleration ${ }^{13,17,20,25,28,29}$, vertical (V) acceleration $^{13,17,20,25,28,29}$, angular velocity on the $\mathrm{AP}^{13,17,26,30}$, $\mathrm{ML}^{17,26}$ or $\mathrm{V}$ axis ${ }^{26}$, gait velocity ${ }^{11,13,20,21-23,25,28,29}$, cadence in steps/ $\min ^{13,23,24,29}$, swing duration, stance duration ${ }^{13,23,28}$, double support duration ${ }^{13,23,24}$, step time, count or length ${ }^{13,21,25,27,29}$ and stride frequency, speed or length ${ }^{11,13,20,24,28}$. A summary of the studies reporting each of these outcome measures is provided in Table 2.

The processing of the gait data occurred simultaneously in all studies and all systems were able to handle the complexity of processing such data in a short time. Algorithms were applied to evaluate the input data from the wearable inertial sensors. All but two studies ${ }^{13,28}$ described the data processing methods precisely as well as the estimation context to which the algorithms were applied.

In all studies, data were collected wirelessly using customized software that automatically provided the variables. The sensor signals were amplified, low pass filtered to remove electronic noise, and transferred to a software program for analysis. A wide range of sampling frequencies was used to assess gait in the studies reviewed, with authors reporting sampling frequencies ranging from 40 to $256 \mathrm{~Hz}$ and configured to collect data at a slower rate $^{11,17,20,21,23-26,29,30}$. Only one study had a frequency set to collect data at a moderate rate ${ }^{22}$. Three studies failed to report the sampling frequency $y^{13,27,28}$. 


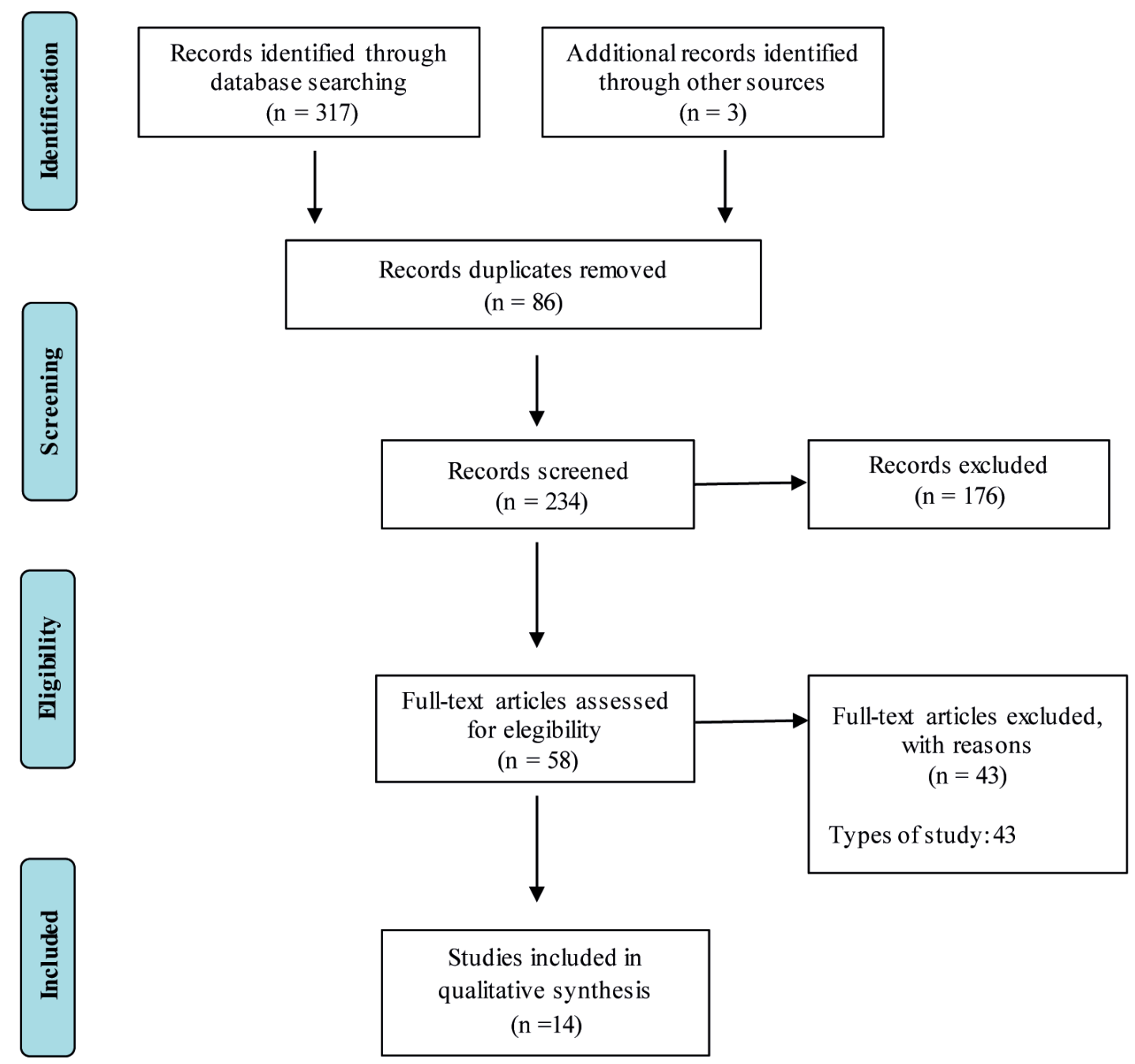

Figure 1 - Flow diagram of the inclusion process of the articles selection procedure.

Table 1 - Methodological quality and reporting of eligible studies.

\begin{tabular}{|c|c|c|c|c|c|c|c|}
\hline \multicolumn{1}{|c|}{ ROBINS-I Tool } \\
\hline \multicolumn{1}{|c|}{ Study } & $\begin{array}{c}\text { Bias due to } \\
\text { confounding }\end{array}$ & $\begin{array}{c}\text { Bias in the } \\
\text { selection of } \\
\text { participants } \\
\text { into the study }\end{array}$ & $\begin{array}{c}\text { Bias in the } \\
\text { classification } \\
\text { of interven- } \\
\text { tions }\end{array}$ & $\begin{array}{c}\text { Bias due to } \\
\text { deviations } \\
\text { from intended } \\
\text { interventions }\end{array}$ & $\begin{array}{c}\text { Bias due to } \\
\text { missing data }\end{array}$ & $\begin{array}{c}\text { Bias in mea- } \\
\text { surement of } \\
\text { outcomes } \\
\text { selection of } \\
\text { the reported } \\
\text { result }\end{array}$ \\
\hline $\begin{array}{l}\text { Antunes } \\
(2016)^{23}\end{array}$ & Serious & Low & Low & Low & Low & Low & Low \\
\hline $\begin{array}{l}\text { Schulleri } \\
(2016)^{21}\end{array}$ & Moderate & Low & Low & Low & Low & Low & Low \\
\hline Suarez $(2016)^{22}$ & Moderate & Low & Low & Low & Low & Low & Low \\
\hline
\end{tabular}


Table 2 - Characteristics of studies included in systematic review $(n=15)$

\begin{tabular}{|c|c|c|c|c|c|}
\hline $\begin{array}{l}\text { Author/year } \\
\text { / study design }\end{array}$ & Participants & $\begin{array}{l}\text { Sensor type } \\
\text { (placement) }\end{array}$ & $\begin{array}{l}\text { Compari- } \\
\text { sons to other } \\
\text { methods }\end{array}$ & Measures & Findings \\
\hline $\begin{array}{c}\text { Mazza }(2010)^{20} \\
\text { Cross-sectional } \\
\text { study }\end{array}$ & $\begin{array}{l}\mathrm{N}=30 \\
15=\text { healthy female } \\
\text { group } \\
\text { (mean age: } 9 \pm 1 \mathrm{yrs} \text { ) } \\
15=\text { healthy male } \\
\text { group } \\
\text { (mean age: } 9 \pm 1 \mathrm{yrs} \text { ) }\end{array}$ & $\begin{array}{l}\text { Three units of } \\
\text { tri-axial acceler- } \\
\text { ometer, triaxial } \\
\text { gyroscope, and } \\
\text { magnetometers } \\
\text { Freq: } 100 \mathrm{~Hz} \\
\text { Sacrum, C7 } \\
\text { intervertebral } \\
\text { space and head }\end{array}$ & $\begin{array}{l}\text { No comparison } \\
\text { performed }\end{array}$ & $\begin{array}{l}\text { Anteroposterior } \\
\text { (AP) acceleration } \\
\text { Mediolateral (ML) } \\
\text { acceleration } \\
\text { Vertical (V) accel- } \\
\text { eration } \\
\text { Stride frequency } \\
\text { Walking Speed }\end{array}$ & $\begin{array}{l}\text { No differences were found } \\
\text { between two groups in pelvis } \\
\text { and shoulder acceleration RMS } \\
\text { values. Conversely, lower head } \\
\text { acceleration RMS values were } \\
\text { found for females in both AP and } \\
\text { ML directions. Both groups man- } \\
\text { aged to attenuate upper body AP } \\
\text { and ML accelerations going from } \\
\text { pelvis-to-head level, with higher } \\
\text { attenuations found for females. }\end{array}$ \\
\hline $\begin{array}{l}\text { Antunes }(2016)^{23} \\
\text { Crossover Trial }\end{array}$ & $\begin{array}{l}\mathrm{N}=20 \\
10 \text { bilateral spastic } \\
\text { cerebral palsy (age: } \\
10.1 \pm 3.7 \text { yrs) } \\
10 \text { healthy children } \\
\text { (age: } 10.3 \pm 4.36 \text { yrs) }\end{array}$ & $\begin{array}{l}\text { One unit of } \\
\text { tri-axial acceler- } \\
\text { ometer, triaxial } \\
\text { gyroscope, and } \\
\text { magnetometers } \\
\text { Freq: } 100 \mathrm{~Hz} \\
\text { L5 intervertebral } \\
\text { space }\end{array}$ & $\begin{array}{l}\text { No comparison } \\
\text { performed }\end{array}$ & $\begin{array}{l}\text { Gait velocity }[\mathrm{cm} / \mathrm{s}] \\
\text { Cadence [steps } / \mathrm{min}] \\
\text { Swing duration [\% of } \\
\text { gait cycle] } \\
\text { Rolling phase [\% of } \\
\text { gait cycle] } \\
\text { Double support } \\
\text { duration [\% of gait } \\
\text { cycle] }\end{array}$ & $\begin{array}{l}\text { Differences were found in both } \\
\text { groups for all spatiotemporal vari- } \\
\text { ables, except for gait velocity. The } \\
\text { percentage of the rolling phase and } \\
\text { double support improved after the } \\
\text { walk-trot task. }\end{array}$ \\
\hline $\begin{array}{l}\text { Schulleri }(2016)^{21} \\
\text { Quasi-experimental } \\
\text { clinical trial }\end{array}$ & $\begin{array}{l}\mathrm{n}=65 \\
26 \text { children and ado- } \\
\text { lescents with spastic } \\
\text { or ataxic cerebral } \\
\text { palsy (age: } 9.8 \pm 4.5 \\
\text { yrs) } \\
39 \text { with typical devel- } \\
\text { opment } \\
\text { (age: } 10.0 \pm 4.4 \text { yrs) }\end{array}$ & $\begin{array}{l}\text { Four units of a } \\
\text { tri-axial acceler- } \\
\text { ometer } \\
\text { Freq: } 60 \mathrm{~Hz} \\
\text { Both lower } \\
\text { legs laterally, } \\
\text { sternum and } \\
\text { forehead }\end{array}$ & $\begin{array}{l}\text { No comparison } \\
\text { performed }\end{array}$ & $\begin{array}{l}\text { Gait speed } \\
\text { Average step } \\
\text { length } \\
\text { Head and trunk } \\
\text { velocity sway }\end{array}$ & $\begin{array}{l}\text { Deliberately light interpersonal } \\
\text { contact applied to the apex of the } \\
\text { head during walking results in the } \\
\text { reduction of head velocity sway, } \\
\text { reducing patterns of spastic or } \\
\text { ataxic movements of the head. }\end{array}$ \\
\hline $\begin{array}{l}\text { Suarez }(2016)^{22} \\
\text { Controlled clinical } \\
\text { trial }\end{array}$ & $\begin{array}{l}\mathrm{n}=24 \\
(10-16 \text { years old }) \\
10 \text { children using } \\
\text { prelingual cochlear } \\
\text { implants } \\
14 \text { children with } \\
\text { normal hearing }\end{array}$ & $\begin{array}{l}\text { Three units of } \\
\text { tri-axial accel- } \\
\text { erometers and } \\
\text { gyroscopes } \\
\text { Freq: } 256 \mathrm{~Hz} \\
\text { Sacrum and on } \\
\text { metatarsal of } \\
\text { each foot }\end{array}$ & $\begin{array}{l}\text { No comparison } \\
\text { performed }\end{array}$ & Gait velocity & $\begin{array}{l}\text { Acoustic information generates } \\
\text { slower gait in those implanted after } 3 \\
\text { years of age. Gait velocity was lower } \\
\text { during a dual task in children with } \\
\text { normal hearing than with children } \\
\text { using prelingual cochlear implants. }\end{array}$ \\
\hline $\begin{array}{l}\text { Bisi }(2017)^{17} \\
\text { Cross-sectional } \\
\text { stud }\end{array}$ & $\begin{array}{l}\mathrm{n}=45 \\
\text { Children with typical } \\
\text { development aged } \\
6-10 \text { yrs; } 3 \text { groups } \\
\text { of } 15 \text { children each } \\
\text { divided by age: } \\
6 Y C=6 \text {-year-old } \\
\text { children } \\
8 Y C=8 \text {-year-old } \\
\text { children } \\
10 Y C=10 \text {-year-old } \\
\text { children }\end{array}$ & $\begin{array}{l}\text { One unit } \\
\text { of tri-axial } \\
\text { accelerometer, } \\
\text { gyroscope, and } \\
\text { magnetometers } \\
\text { Freq: } 128 \mathrm{~Hz} \\
\text { Lower back, } \\
\text { ankles (above } \\
\text { lateral malleo- } \\
\text { lus) and wrists }\end{array}$ & $\begin{array}{l}\text { Standard } \\
\text { assessment } \\
\text { based on } \\
\text { video-record- } \\
\text { ings by expert } \\
\text { operators }\end{array}$ & $\begin{array}{l}\text { (AP) acceleration } \\
\text { Mediolateral (ML) } \\
\text { acceleration } \\
\text { Vertical (V) accel- } \\
\text { eration } \\
\text { Angular velocity on } \\
\text { AP axis ( } \omega \mathrm{AP}) \\
\text { Angular velocity on } \\
\text { ML axis }(\omega \mathrm{ML})\end{array}$ & $\begin{array}{l}\text { Automatic assessment based } \\
\text { on wearable IMUs compared } \\
\text { to standard assessment showed } \\
\text { agreement higher than } 87 \% \text { on } \\
\text { average in the entire group for } \\
\text { each skill and reduction in time } \\
\text { for scoring from } 15 \text { to } 2 \text { minutes } \\
\text { per participant. }\end{array}$ \\
\hline
\end{tabular}




\begin{tabular}{|c|c|c|c|c|c|}
\hline $\begin{array}{l}\text { Author/year } \\
\text { / study design }\end{array}$ & Participants & $\begin{array}{l}\text { Sensor type } \\
\text { (placement) }\end{array}$ & $\begin{array}{l}\text { Compari- } \\
\text { sons to other } \\
\text { methods }\end{array}$ & Measures & Findings \\
\hline $\begin{array}{l}\text { Ganea. }(2012)^{24} \\
\text { Cross-sectional } \\
\text { study }\end{array}$ & $\begin{array}{l}\mathrm{n}=45 \\
\text { Enrolled in two } \\
\text { groups: } \\
25 \text { ambulatory boys } \\
\text { with Duchenne mus- } \\
\text { cular dystrophy aged } \\
5 \text { to } 12 \text { years }(8.04 \\
\pm 1.9) \\
20 \text { age-matched } \\
\text { ( } 7.85 \pm 2.48) \text { healthy } \\
\text { children }(12 \text { boys and } \\
8 \text { girls) }\end{array}$ & $\begin{array}{l}\text { One unit of } \\
\text { uniaxial gyro- } \\
\text { scope and } \\
\text { tri-axial accel- } \\
\text { erometer } \\
\text { Freq: } 40 \mathrm{~Hz} \\
\text { Shanks }\end{array}$ & $\begin{array}{l}\text { No comparison } \\
\text { performed }\end{array}$ & $\begin{array}{l}\text { Stride length (SL) } \\
\text { Shank peak angular } \\
\text { velocity (SPAV) } \\
\text { Stride velocity (SV) } \\
\text { Cadence (Cad) } \\
\text { Double support } \\
\text { (DS) }\end{array}$ & $\begin{array}{l}\text { Compared with healthy children, } \\
\text { patients with Duchenne muscular dys- } \\
\text { trophy had significantly lower stride } \\
\text { velocity and less smooth trunk move- } \\
\text { ment. When a group of patients was } \\
\text { divided into mild and moderate based } \\
\text { on Motor Function Measure, authors } \\
\text { noticed significantly higher values both } \\
\text { for cadence and stride velocity as well } \\
\text { as improved trunk smoothness in mild } \\
\text { versus moderate group. Potential of } \\
\text { such variables to distinguish between } \\
\text { different disease states opens new } \\
\text { perspectives for objective assessments } \\
\text { of effectiveness of new therapies for } \\
\text { Duchenne muscular dystrophy }\end{array}$ \\
\hline $\begin{array}{l}\text { Summa }(2016)^{25} \\
\text { Cross-sectional } \\
\text { study }\end{array}$ & $\begin{array}{l}\mathrm{n}=40 \\
20 \text { children with } \\
\text { cerebral palsy } \\
\text { (Age } 5.70 \pm 2.27 \\
\text { years, range } 2-9 \\
\text { years) } \\
20 \text { children with } \\
\text { Typically developing } \\
\text { (Age } 5.85 \pm 2.18 \\
\text { years, range } 2-9 \\
\text { years) }\end{array}$ & $\begin{array}{l}\text { Three units } \\
\text { of tri-axial } \\
\text { accelerometer, } \\
\text { gyroscope, and } \\
\text { magnetometers } \\
\text { Freq: } 128 \mathrm{~Hz} \\
\text { Head level } \\
\text { (occipital } \\
\text { cranium bone), } \\
\text { sternum level } \\
\text { and on pelvis } \\
\text { (sacrum-L5 } \\
\text { level) }\end{array}$ & $\begin{array}{l}\text { No comparison } \\
\text { performed }\end{array}$ & $\begin{array}{l}\text { Anteroposterior } \\
\text { (AP) acceleration } \\
\text { Mediolateral (ML) } \\
\text { acceleration } \\
\text { Vertical (V) accel- } \\
\text { eration } \\
\text { Step length } \\
\text { Step frequency } \\
\text { Walking speed }\end{array}$ & $\begin{array}{l}\text { Despite a significant reduction in } \\
\text { acceleration from the pelvis to the } \\
\text { sternum, children with cerebral } \\
\text { palsy do not compensate for large } \\
\text { accelerations, which are greater } \\
\text { than in children with typical devel- } \\
\text { opment. The children with cerebral } \\
\text { palsy had negative sternum-to- } \\
\text { head attenuations in agreement } \\
\text { with documented rigidity of the } \\
\text { head-trunk system observed in this } \\
\text { population. }\end{array}$ \\
\hline $\begin{array}{l}\text { Chen }(2017)^{26} \\
\text { Cross-sectional } \\
\text { study }\end{array}$ & $\begin{array}{l}\mathrm{n}=46 \\
14 \text { Healthy adults } \\
(24.2 \pm 1.55 \text { years }) \\
10 \text { Healthy children } \\
\text { (7.03 } \pm 1.49 \text { years }) \\
22 \text { Children with } \\
\text { cerebral palsy } \\
(7.51 \pm 2.96 \text { years })\end{array}$ & $\begin{array}{l}\text { Three units of } \\
\text { tri-axial accel- } \\
\text { erometers } \\
\text { and gyroscopes } \\
\text { Freq: } 100 \mathrm{~Hz} \\
\text { Lower back } \\
\text { (L2-L3) and on } \\
\text { the middle of } \\
\text { right and left } \\
\text { thigh (semiten- } \\
\text { dinosus) }\end{array}$ & $\begin{array}{l}\text { No comparison } \\
\text { performed }\end{array}$ & $\begin{array}{l}\text { Anteroposterior } \\
\text { (AP) acceleration } \\
\text { Mediolateral (ML) } \\
\text { acceleration } \\
\text { Vertical (V) accel- } \\
\text { eration } \\
\text { Angular velocity on } \\
\text { AP, ML and V axes }\end{array}$ & $\begin{array}{l}\text { Compared with healthy subjects, } \\
\text { symptoms and severity of motor } \\
\text { dysfunction in cerebral palsy chil- } \\
\text { dren could result in abnormality of } \\
\text { gait acceleration modes, and the pro- } \\
\text { posed assessment method was able } \\
\text { to effectively evaluate the degree } \\
\text { of gait abnormality in children with } \\
\text { cerebral palsy. }\end{array}$ \\
\hline $\begin{array}{l}\text { Zollinger (2016) } \\
\text { Cross-sectional } \\
\text { study }\end{array}$ & $\begin{array}{l}\mathrm{n}=20 \\
10 \text { unilateral cerebral } \\
\text { palsy } \\
(14.2 \pm 1.7 \text { years }) \\
10 \text { typically devel- } \\
\text { oping } \\
(14.1 \pm 1.9 \text { years })\end{array}$ & $\begin{array}{l}\text { Two units of } \\
\text { tri-axial accel- } \\
\text { erometers and } \\
\text { gyroscopes } \\
\text { Freq: } 100 \mathrm{~Hz} \\
\text { Lower part } \\
\text { of back, (L3 } \\
\text { vertebra region) } \\
\text { and on instep of } \\
\text { foot of subject }\end{array}$ & $\begin{array}{l}\text { No other } \\
\text { comparison } \\
\text { performed }\end{array}$ & $\begin{array}{l}\text { Mass center accel- } \\
\text { eration } \\
\text { Three dimensional } \\
\text { accelerations of foot }\end{array}$ & $\begin{array}{l}\text { Evaluation of inertial sensor gait } \\
\text { pattern revealed that treadmill } \\
\text { training induced mechanical changes } \\
\text { almost identical to overground walk- } \\
\text { ing in both groups. with exception } \\
\text { of potential and kinetic vertical and } \\
\text { lateral mechanical works, which are } \\
\text { both significantly increased in over- } \\
\text { ground - treadmill transition only in } \\
\text { unilateral cerebral palsy. }\end{array}$ \\
\hline $\begin{array}{l}\text { Christensen } \\
(2017)^{27} \\
\text { Cross-sectional } \\
\text { study }\end{array}$ & $\begin{array}{l}\mathrm{n}=75 \\
\text { Children with diagno- } \\
\text { sis of idiopathic toe } \\
\text { walking aged } 3-13 \\
\text { years; divided into } \\
\text { two groups by age: } \\
\quad \text { 2-to-5-y-olds }= \\
\text { 456-to-13-y-olds } \\
\quad=30\end{array}$ & $\begin{array}{l}\text { One unit of } \\
\text { uni-axial accel- } \\
\text { erometer } \\
\text { Freq: Not } \\
\text { reported } \\
\text { Waist }\end{array}$ & $\begin{array}{l}\text { Video obser- } \\
\text { vation }\end{array}$ & Step counts & $\begin{array}{l}\text { Significant difference in accelerom- } \\
\text { eter scores and test pitch for children } \\
2 \text { to } 5 \text { years old; no significant differ- } \\
\text { ence found among } 6 \text {-to- } 13 \text {-year-olds. }\end{array}$ \\
\hline
\end{tabular}




\begin{tabular}{|c|c|c|c|c|c|}
\hline $\begin{array}{l}\text { Author/year } \\
\text { / study design }\end{array}$ & Participants & $\begin{array}{l}\text { Sensor type } \\
\text { (placement) }\end{array}$ & $\begin{array}{l}\text { Compari- } \\
\text { sons to other } \\
\text { methods }\end{array}$ & Measures & Findings \\
\hline $\begin{array}{l}\text { Lanovaz }(2017)^{11} \\
\text { Cross-sectional } \\
\text { study }\end{array}$ & $\begin{array}{l}\mathrm{n}=10 \\
\text { Typically developing } \\
\text { children } \\
\text { (mean age: } 5.1 \text { years, } \\
\text { range: } 3.0 \text { to } 8.3 \\
\text { years) }\end{array}$ & $\begin{array}{l}\text { Six units } \\
\text { of tri-axial } \\
\text { accelerometers, } \\
\text { gyroscopes, and } \\
\text { magnetometers } \\
\text { Freq: } 128 \mathrm{~Hz} \\
\text { Dorsal side of } \\
\text { both wrists, } \\
\text { sternum close } \\
\text { to clavicular } \\
\text { notch, lower } \\
\text { back (L4/L5) } \\
\text { and on the } \\
\text { front side of } \\
\text { shins close to } \\
\text { malleoli }\end{array}$ & $\begin{array}{l}\text { 3D motion } \\
\text { capture system }\end{array}$ & $\begin{array}{l}\text { Stride time } \\
\text { Stance time } \\
\text { Stride length } \\
\text { Stride velocity } \\
\text { Walking velocity }\end{array}$ & $\begin{array}{l}\text { All spatiotemporal variables evaluat- } \\
\text { ed showed good agreement between } \\
\text { the two systems. }\end{array}$ \\
\hline $\begin{array}{l}\text { Pendharkar } \\
(2012)^{28} \\
\text { Cross-sectional } \\
\text { study }\end{array}$ & $\begin{array}{l}\mathrm{n}=20 \\
\text { (mean age: } 8 \text { years; } \\
\text { mean weight: } 25 \mathrm{~kg} \text { ) } \\
10 \text { healthy children. } \\
10 \text { children with idio- } \\
\text { pathic toe walking }\end{array}$ & $\begin{array}{l}\text { One unit of } \\
\text { dual-axis accel- } \\
\text { erometer } \\
\text { Freq: Not } \\
\text { reported } \\
\text { Heel of boot }\end{array}$ & $\begin{array}{l}\text { No comparison } \\
\text { performed }\end{array}$ & \begin{tabular}{l|} 
Stance phase \\
Swing phase \\
Number of strides \\
Walking speed \\
Vertical acceleration \\
Horizontal acceler- \\
ation \\
Gravitational accel- \\
eration
\end{tabular} & $\begin{array}{l}\text { Foot angle during mid-stance ranged } \\
\text { from } 36^{\circ} \text { to } 11.5^{\circ} \text { in children with } \\
\text { idiopathic toe walking, but foot } \\
\text { stance angle was approximately zero } \\
\text { in normal children. }\end{array}$ \\
\hline $\begin{array}{l}\text { Saether }(2014)^{29} \\
\text { Cross-sectional } \\
\text { study }\end{array}$ & $\begin{array}{l}\mathrm{n}=70 \\
41 \text { spastic cerebral } \\
\text { palsy } \\
(11.7 \pm 3.8 \text { years }) \\
29 \text { typically develop- } \\
\text { ing children } \\
(10.3 \pm 3.6 \\
\text { years })\end{array}$ & $\begin{array}{l}\text { One unit } \\
\text { of tri-axial } \\
\text { accelerometer, } \\
\text { gyroscope, and } \\
\text { magnetometer } \\
\text { Freq: } 100 \mathrm{~Hz} \\
\text { Lower back } \\
\text { (over L3 } \\
\text { region) }\end{array}$ & $\begin{array}{l}\text { No comparison } \\
\text { performed }\end{array}$ & $\begin{array}{l}\text { Trunk acceleration } \\
\text { (Anteroposterior } \\
\text { (AP), Vertical (V) } \\
\text { and Mediolateral } \\
\text { (ML) } \\
\text { Gait speed } \\
\text { Cadence } \\
\text { Step time } \\
\text { Step length }\end{array}$ & $\begin{array}{l}\text { Gait variables related to balance (AP, } \\
\text { ML, and V accelerations) were higher in } \\
\text { children with CP and increased with an } \\
\text { increase in GMFCS level. Differences } \\
\text { in acceleration in AP and V directions } \\
\text { increased between children with CP } \\
\text { and TD children with increase in speed. } \\
\text { Asymmetry in trunk acceleration differed } \\
\text { significantly between two groups in } \\
\text { all three directions (z-scores between } \\
0.8 \text { and } 1.8 \text { higher in CP group), while } \\
\text { inter-stride regularity differed only } \\
\text { slightly between children with CP and } \\
\text { TD children and only in AP direction. } \\
\text { Gait characteristics also differed between } \\
\text { children with unilateral and bilateral } \\
\text { spastic subtypes of CP for acceleration } \\
\text { and asymmetry in AP and ML directions. }\end{array}$ \\
\hline $\begin{array}{l}\text { Sivarajah }(2017)^{13} \\
\text { Cross-sectional } \\
\text { study }\end{array}$ & $\begin{array}{l}\mathrm{n}=30 \\
15 \text { children with spi- } \\
\text { na bifida or cerebral } \\
\text { palsy (mean age: } 7.9 \\
\pm 3.1 \text { years, } 8 \text { males) } \\
15 \text { typically develop- } \\
\text { ing children (mean } \\
\text { age: } 8.2 \pm 3.2 \text { years, } \\
8 \text { males) }\end{array}$ & $\begin{array}{l}\text { Six units } \\
\text { of tri-axial } \\
\text { accelerometer, } \\
\text { gyroscopes and } \\
\text { magnetometers } \\
\text { Freq: Not } \\
\text { reported } \\
\text { One sensor on } \\
\text { each ankle and } \\
\text { wrist, one on } \\
\text { lower back and } \\
\text { one on upper } \\
\text { chest }\end{array}$ & $\begin{array}{l}\text { No comparison } \\
\text { performed }\end{array}$ & $\begin{array}{l}\text { Stride length ROM of } \\
\text { trunk on horizontal, } \\
\text { sagittal, and frontal } \\
\text { planes (degrees) } \\
\text { Peak angular velocity } \\
\text { of trunk on sagittal } \\
\text { plane } \\
\text { Peak velocity of } \\
\text { trunk on horizontal, } \\
\text { sagittal and frontal } \\
\text { planes (degrees/ } \\
\text { second) } \\
\text { Cadence (steps/min) } \\
\text { Double support } \\
\text { (percentage of gait } \\
\text { cycle) } \\
\text { Swing and stance } \\
\text { asymmetry } \\
\text { Number of steps }\end{array}$ & $\begin{array}{l}\text { On 10-Meter Walk Test, group } \\
\text { differences were found in horizontal } \\
\text { and frontal trunk range of motion, } \\
\text { horizontal trunk velocity and swing } \\
\text { asymmetry. Children with spina } \\
\text { bifida or cerebral palsy took signifi- } \\
\text { cantly longer to turn during Timed } \\
\text { Up and Go Test. These five variables } \\
\text { together distinguished the groups. }\end{array}$ \\
\hline
\end{tabular}




\begin{tabular}{|l|l|l|l|l|l|}
\hline \multicolumn{1}{|c|}{$\begin{array}{c}\text { Author/year } \\
\text { / study design }\end{array}$} & \multicolumn{1}{|c|}{ Participants } & $\begin{array}{c}\text { Sensor type } \\
\text { (placement) }\end{array}$ & $\begin{array}{c}\text { Compari- } \\
\text { sons to other } \\
\text { methods }\end{array}$ & \multicolumn{1}{|c|}{ Measures } & Findings \\
\hline $\begin{array}{l}\text { Taborri }(2015)^{30} \\
\text { Cross-sectional } \\
\text { study }\end{array}$ & $\begin{array}{l}\mathrm{n}=20 \\
10 \text { children with } \\
\text { hemiplegia } \\
(7.8 \pm 2.8 \text { years) } \\
10 \text { children with typi- } \\
\text { cal development } \\
(9.5 \pm 2.0 \text { years) }\end{array}$ & $\begin{array}{l}\text { Two units of } \\
\text { uni-axial gyro- } \\
\text { scopes } \\
\text { Freq: } 50 \mathrm{~Hz} \\
\text { Foot and shin } \\
\text { of the dominant } \\
\text { leg for typically } \\
\text { developing } \\
\text { children and } \\
\text { on the more } \\
\text { affected leg } \\
\text { for hemiplegic } \\
\text { children }\end{array}$ & $\begin{array}{l}\text { No comparison } \\
\text { performed }\end{array}$ & $\begin{array}{l}\text { Angular velocities } \\
\text { on sagittal plane of } \\
\text { shin and foot }\end{array}$ & $\begin{array}{l}\text { Adequacy of classifiers was evaluat- } \\
\text { ed using receiver operating charac- } \\
\text { teristics. Good to optimum results } \\
\text { for all classifiers examined, with the } \\
\text { best performance for the distributed } \\
\text { classifier in two-phase recognition. } \\
\text { Differences were found between gait } \\
\text { partitioning models, while no differ- } \\
\text { ences were found between training } \\
\text { procedures with the exception of } \\
\text { shin classifier. }\end{array}$ \\
\hline
\end{tabular}

Abbreviations: YC: year-old children; IMUs: inertial measurement units; Freq: sampling frequency of wearable sensor; ROM: range of motion

\section{Discussion}

Based on the appraisal of the methodological quality of the articles included, overall scientific reporting in this field is largely of adequate quality. However, the information united in this review was produced by different experimental designs, which leads to distinct data. The papers lacked details concerning the representativeness of the study population, the approaches adopted to identify and account for confounding variables, and appropriate justification for the chosen sample size. This does not mean that the authors did not consider some or all of these factors, but rather suggests that these aspects require greater attention in the reporting of future research.

All studies reported accuracy in the gait evaluations using wearable inertial sensors. However, only three studies compared their results to other techniques, such as a video-based motion capture system ${ }^{11,17}$. The detection of accuracy using algorithms for inertial measurement units with a gyroscope and magnometer was higher than that using algorithms based on accelerometers alone. None of the studies reported measurements errors associated during the analysis of lower limb movements.

The choice of the sensor was not correlated with either the variables evaluated or the application. The sensors were mostly placed on the lower limbs. The choice of the body region for the placement of a sensor is of extreme importance to the expected objective of the analysis. The literature reports a variety of variables collected using inertial sensor-based motion analysis, such as angular and temporal variables of the trunk, upper limbs, and head, which are used to quantify various movement disorders, such as trunk control, balance and angular position of the head and arms during gait ${ }^{32,33}$. In summary, there are no clinical practice guidelines for reporting the application of the use of inertial sensors, which hinders the comparison of results from different studies and overcoming problems that may occur (such as data processing or the study of biomechanical variables).

There are very few reports of difficulties during the test protocols performed with inertial sensors. The majority of children were willing to wear the inertial sensors during the gait assessments. In only one study ${ }^{13}$, a two-year-old child with cerebral palsy did not want to use the sensors and therefore did not complete the study, but no explanation of why was given. It, therefore, appears that inertial sensors constitute a feasible tool for gait evaluations in the pediatric population and can be attached to different body segments. The degree of accuracy and reliability reported in the studies included in this review suggests that these sensors can be used for the repeated measurement of specific movements in different contexts, such as non-hospital and non-laboratory settings (private homes or clinics) $)^{11,13,17,20,21,23-25,27-29}$ with a significant advantage associated with the unbiased results compared to the qualitative estimates of a therapist ${ }^{32}$. Inertial sensors can also be used to complement gold standard measures (multi-camera motion capture system and force plate).

The application prospects of wearable inertial sensors in different types of pediatric conditions were explored in this review, as we can see in table 2 . Such sensors constitute a useful tool in both clinical practice and biomechanical research involving children with typical development and those with neurological and/or muscular diseases, such as muscular dystrophy and cerebral palsy. The experimental results of the studies analyzed herein suggest that wearable sensors are effective for the evaluation of quantitative gait variables in children with different pediatric conditions, enabling an objective analysis that should prove useful in processes of clinical diagnosis and rehabilitation. Mannini et $\mathrm{al}^{33}$ demonstrated that automatic classification employing signals from inertial sensors obtained during gait can also be used as a support tool in the differential diagnosis, assisting in improving diagnostic accuracy in cases of coordination impairment in children.

Several limitations should be considered when interpreting the results of this review of the literature. Firstly, given the relatively small number of studies published on this topic, it is difficult to make strong recommendations regarding the most appropriate equipment, sensor placement, and outcomes for assessing gait in children. Second, the outcomes of this systematic review show a lack of standardization in reporting the methods 
and results of evaluations involving adaptive algorithms for the determination of spatiotemporal gait variables. Such aspects need to be evaluated given the encouraging results of the papers. Third, this systematic review did not address the reliability of analytical algorithms for gait kinetics. Future studies should give careful consideration to the internal and external validity of the methods employed as well as the detection accuracy and delay of the different types of wearable sensors used in clinical practice.

\section{Conclusion}

Wearable sensors are potentially useful for the study of gait patterns in children, including compensatory patterns, and can be used to support therapists and physicians in the design of innovative intervention protocols and monitoring the effectiveness of such protocols in terms of improvements in gait. Wearable sensors constitute a light-weight, portable, affordable alternative to more expensive three-dimensional motion analysis systems and are effective at detecting changes in children's gait.

\section{References}

1. Ghoussayni S, Stevens C, Durham S, Ewins D. Assessment and validation of a simple automated method for the detection of gait events and intervals. Gait Posture. 2004; 20: 266-272.

2. Worsley PR, Whatling G, Barrett D, Holt C, Stokes M, Taylor M. Assessing changes in subjective and objective function from pre-to post-knee arthroplasty using the Cardiff Dempster-Shafer Theory classifier. Comput Methods Biomech Biomed Engin. 2015; 22:1-10. doi: 10.1080/10255842.

3. Ganley KJ, Powers CM. Gait kinematics and kinetics of 7-yearold children: a comparison to adults using age-specific anthropometric data. Gait Posture. 2005; 21:141-145.

4. Kim CM, Eng JJ. Magnitude and pattern of 3D kinematic and kinetic gait profiles in persons with stroke: Relationship to walking speed. Gait Posture. 2004; 20: 140-146.

5. Casadio M, Morasso PG, Sanguineti V. Direct measurement of ankle stiffness during quiet standing: Implications for control modelling and clinical application. Gait Posture. 2005; 21 : 410-424.

6. Komnik I, Weiss S, Pagani CF, Potthast W. Motion analysis of patients after knee arthroplasty during activities of daily living a systematic review. Gait Posture. 2015; 41(2): 370-377. doi:10.1016/j.gaitpost.2015.01.019.

7. Auvinet B, Berrut G, Touzard C, Moutel L, Collet N, Chaleil $\mathrm{D}$, et al. Reference data for normal subjects obtained with an accelerometric device. Gait Posture. 2002; 16:124-34.

8. Tao W, Liu T, Zheng R, Feng H. Gait analysis using wearable sensors. Sensors. 2012; 12: 2255-2283.

9. Boutaayamou M, Schwartz C, Stamatakis J, Denoel V, Maquet D, Forthomme B et al. Development and validation of an accelerometer-based method for quantifying gait events. Med. Eng. Phys. 2015; 37: 226-232. doi:10.1016/S1350-4533(03)00116-4.

10. Shull PB, Jirattigalachote W, Hunt MA, Cutkosky, Delp SL. Quantified self and human movement: a review on the clinical impact of wearable sensing and feedback for gait analysis and intervention, Gait Posture. 2014; 40: 11-19.

11. Lanovaz JL, Oates AR, Treen TT, Unger J. Musselman, K.E. Validation of a commercial inertial sensor system for spatiotemporal gait measurements in children. Gait Posture. 2017; 51: 14-19.

12. Pimentel R, Sauer C, Carollo J. Validation of Gait Cycle Timing Using Wearable Sensors in Individuals with Cerebral Palsy. Arch Phys Med Rehabil. 2017; 98(10): 129-e130.

13. Sivarajah L, Kane KJ, Lanovaz J, Bisaro D, Oates A, Ye M, et al. The Feasibility and Validity of Body-Worn Sensors to Supplement Timed Walking Tests for Children with Neurological Conditions. Phys Occup Ther Pediatr.2017; 38(3):280-290.

14. Schwesig R, Leuchte S, Fischer D, Ullmann R, Kluttig A. Inertial sensor-based reference gait data for healthy subjects. Gait posture.2011; 33(4): 673-678.

15. Mariani B, Hoskovec C, Rochat S, Bula C, Penders J, Aminian K. $3 \mathrm{D}$ gait assessment in young and elderly subjects using foot-worn inertial sensors. J Biomech. 2010; 43(15):2999-3006.

16. Mariani B, Jimenez MC, Vingerhoets FJ, Aminian K. On-shoe wearable sensors for gait and turning assessment of patients with Parkinson's disease. IEEE Trans Biomed Eng. 2013;60(1):155-8.

17. Bisi MC, Pasini PG, Polman R, Stagni R. Objective assessment of movement competence in children using wearable sensors: An instrumented version of the TGMD-2 locomotor subtest. Gait Posture. 2017;56:42-48. doi: 10.1016/j.gaitpost.2017.04.025.

18. Sterne JAC, Hernán MA, Reeves BC, Savovic J, Berkman ND, Viswanathan, M, et al. ROBINS-I: a tool for assessing the risk of bias in non-randomized studies of interventions. BMJ. 2016;355:i4919.

19. Downes MJ, Brennan ML, Williams HC, Dean RS. Development of a critical appraisal tool to assess the quality of cross-sectional studies (AXIS). BMJ Open. 2016;6:e011458.doi:10.1136/ bmjopen-2016-011458.

20. Mazzà C, Zok M, Cappozzo A. Head stabilization in children of both genders during level walking. Gait Posture. 2010; 31:429-432.

21. Schulleri KH, Burfeind F, Höß-Zenker B, Szabó ÉF, Herzig N, Ledebt A, et al. Deliberately light interpersonal contact affects the control of head stability during walking in children and adolescents with cerebral palsy. Archives of Physical Medicine and Rehabilitation, 2017; 98: 1828-1835. doi:10.1016/j. apmr.2017.01.026.

22. Suarez H, Alonso R, Arocena S, Ferreira E, San Roman C, Suarez A, et al. Sensory-motor interaction in deaf children. Relationship between gait performance and hearing input during childhood assessed in prelingual cochlear implant users. Acta Otolaryngol. 2016; 15:1-6.

23. Antunes FN, Pinho AS, Kleiner AFR, Salazar AP, Eltz GD, de Oliveira Junior AA, Cechettim et al. Different horse's paces during hippotherapy on spatiotemporal parameters of gait in children with bilateral spastic cerebral palsy: A feasibility study. Res Dev Disabil. 2016; 59:65-72.

24. Ganea R, Jeannet PY, Paraschiv-Ionescu A, Goemans NM, Piot C, Van den Hauwe, et al. Gait assessment in children with Duchenne muscular dystrophy during long-distance walking. J. Child Neurol. 2012; 27(1): 30-38. 
25. Summa A, Vannozzi G, Bergamini E, Iosa M, Morelli D, Cappozzo A. Multilevel upper body movement control during gait in children with cerebral palsy. PLoS One. 2016;11(3): e0151792.

26. Chen X, Liao S, Cao S, Wu D, Zhang X. An Acceleration-Based Gait Assessment Method for Children with Cerebral Palsy. Sensors.2017; 17(5): 1002.

27. Christensen C, Haddad A, Maus E. The validation of an accelerometer used to measure step count in children with idiopathic toe walking. Pediatr Phys Ther. 2017;29(2):153-157. doi: 10.1097/ PEP.0000000000000364.

28. Pendharkar G, Percival P, Morgan D, Lai D. Automated method to distinguish toe walking strides from normal strides in the gait of idiopathic toe walking children from heel accelerometry data. Gait Posture. 2012; 35(3):478-82.

29. Saether R, Helbostad JL, Adde L, Brændvik S, Lydersen S, Vik T. Gait characteristics in children and adolescents with cerebral palsy assessed with a trunk-worn accelerometer. Res Dev Disabil. 2014; 35: 1773-81.
30. Taborri J, Scalona E, Palermo E, Rossi S, Cappa P. Validation of Inter-Subject Training for Hidden Markov Models Applied to Gait Phase Detection in Children with Cerebral Palsy. Sensors. 2015; 15: 24514-24529.

31. Sæther R, Helbostad JL, Adde L, Braendvik S, Lydersen S, Vik T. The relationship between trunk control in sitting and during gait in children and adolescents with cerebral palsy. Dev Med Child. 2015; 57:344-50.

32. Sun T, Li H, Liu Q, Duan L, Li M, Wang C et al. Inertial Sensor-Based Motion Analysis of Lower Limbs for Rehabilitation Treatments. J Healthc Eng. 2017;1-11. doi: 10.1155/2017/1949170.

33. Mannini A, Martinez-Manzanera O, Lawerman TF, Trojaniello D, Croce UD, Sival DA, et al. Automatic classification of gait in children with early-onset ataxia or developmental coordination disorder and controls using inertial sensors. Gait Posture. 2017; Feb(52):287-292.

\section{Appendix}

a. AXIS tool for the critical appraisal of Cross-sectional studies.

\begin{tabular}{|c|c|c|c|c|c|c|c|c|c|c|c|c|c|c|c|c|c|c|c|c|}
\hline \multicolumn{21}{|c|}{ Axis Tool $^{\mathrm{a}}$} \\
\hline Study & 1 & 2 & 3 & 4 & 5 & 6 & 7 & 8 & 9 & 10 & 11 & 12 & 13 & 14 & 15 & 16 & 17 & 18 & 19 & 20 \\
\hline Bisi $(2017)^{17}$ & $\mathrm{Y}$ & $\mathrm{Y}$ & $\mathrm{Y}$ & $\mathrm{Y}$ & $\mathrm{Y}$ & $\mathrm{Y}$ & $\mathrm{Y}$ & $\mathrm{Y}$ & $\mathrm{Y}$ & $\mathrm{Y}$ & $\mathrm{Y}$ & $\mathrm{Y}$ & $\mathrm{N}$ & $\mathrm{Y}$ & $\mathrm{Y}$ & $\mathrm{Y}$ & $\mathrm{Y}$ & $\mathrm{Y}$ & $\mathrm{N}$ & $\mathrm{Y}$ \\
\hline Ganea $(2012)^{24}$ & $\mathrm{Y}$ & $\mathrm{Y}$ & $\mathrm{N}$ & $\mathrm{Y}$ & $\mathrm{Y}$ & $\mathrm{Y}$ & $\mathrm{Y}$ & $\mathrm{Y}$ & $\mathrm{Y}$ & $\mathrm{Y}$ & $\mathrm{Y}$ & $\mathrm{Y}$ & $\mathrm{N}$ & $\mathrm{Y}$ & $\mathrm{Y}$ & $\mathrm{Y}$ & $\mathrm{Y}$ & $\mathrm{Y}$ & $\mathrm{N}$ & $\mathrm{Y}$ \\
\hline Summa $(2016)^{25}$ & $\mathrm{Y}$ & $\mathrm{Y}$ & $\mathrm{Y}$ & $\mathrm{Y}$ & $\mathrm{Y}$ & $\mathrm{Y}$ & $\mathrm{Y}$ & $\mathrm{Y}$ & $\mathrm{Y}$ & $\mathrm{Y}$ & $\mathrm{Y}$ & $\mathrm{Y}$ & $\mathrm{N}$ & $\mathrm{Y}$ & $\mathrm{Y}$ & $\mathrm{Y}$ & $\mathrm{Y}$ & $\mathrm{N}$ & $\mathrm{N}$ & $\mathrm{Y}$ \\
\hline Chen $(2017)^{26}$ & $\mathrm{Y}$ & $\mathrm{Y}$ & $\mathrm{N}$ & $\mathrm{Y}$ & $\mathrm{Y}$ & $\mathrm{Y}$ & $\mathrm{Y}$ & $\mathrm{Y}$ & $\mathrm{Y}$ & $\mathrm{Y}$ & $\mathrm{Y}$ & $\mathrm{Y}$ & $\mathrm{N}^{\prime}$ & $\mathrm{Y}$ & $\mathrm{Y}$ & $\mathrm{Y}$ & $\mathrm{Y}$ & $\mathrm{Y}$ & $\mathrm{N}$ & $\mathrm{Y}$ \\
\hline Christensen $(2017)^{27}$ & Y & $\mathrm{N}$ & $\mathrm{Y}$ & $\mathrm{Y}$ & $\mathrm{Y}$ & $\mathrm{Y}$ & $\mathrm{Y}$ & $\mathrm{Y}$ & $\mathrm{Y}$ & $\mathrm{Y}$ & $\mathrm{Y}$ & $\mathrm{Y}$ & $\mathrm{N}$ & $\mathrm{Y}$ & $\mathrm{Y}$ & $\mathrm{Y}$ & $\mathrm{Y}$ & $\mathrm{Y}$ & $\mathrm{N}$ & $\mathrm{Y}$ \\
\hline Lanovaz $(2017)^{11}$ & $\mathrm{Y}$ & $\mathrm{N}$ & $\mathrm{N}$ & $\mathrm{Y}$ & $\mathrm{Y}$ & $\mathrm{Y}$ & $\mathrm{Y}$ & $\mathrm{Y}$ & $\mathrm{Y}$ & $\mathrm{Y}$ & $\mathrm{Y}$ & $\mathrm{Y}$ & $\mathrm{N}$ & $\mathrm{Y}$ & $\mathrm{Y}$ & $\mathrm{Y}$ & $\mathrm{Y}$ & $\mathrm{Y}$ & $\mathrm{N}$ & $\mathrm{Y}$ \\
\hline Pendharkar $(2012)^{28}$ & $\mathrm{Y}$ & $\mathrm{Y}$ & $\mathrm{Y}$ & $\mathrm{Y}$ & $\mathrm{Y}$ & $\mathrm{Y}$ & $\mathrm{Y}$ & $\mathrm{Y}$ & $\mathrm{Y}$ & $\mathrm{Y}$ & $\mathrm{Y}$ & $\mathrm{Y}$ & $\mathrm{N}$ & $\mathrm{Y}$ & $\mathrm{Y}$ & $\mathrm{Y}$ & $\mathrm{Y}$ & $\mathrm{N}$ & $\mathrm{N}$ & $\mathrm{Y}$ \\
\hline Saether $(2014)^{29}$ & $\mathrm{Y}$ & $\mathrm{Y}$ & $\mathrm{Y}$ & $\mathrm{Y}$ & $\mathrm{Y}$ & $\mathrm{Y}$ & $\mathrm{Y}$ & $\mathrm{Y}$ & $\mathrm{Y}$ & $\mathrm{Y}$ & $\mathrm{Y}$ & $\mathrm{Y}$ & $\mathrm{N}$ & $\mathrm{Y}$ & $\mathrm{Y}$ & $\mathrm{Y}$ & $\mathrm{Y}$ & $\mathrm{Y}$ & $\mathrm{N}$ & $\mathrm{Y}$ \\
\hline Sivarajah $(2017)^{13}$ & $\mathrm{Y}$ & $\mathrm{Y}$ & $\mathrm{Y}$ & $\mathrm{Y}$ & $\mathrm{Y}$ & $\mathrm{Y}$ & $\mathrm{Y}$ & $\mathrm{Y}$ & $\mathrm{Y}$ & $\mathrm{Y}$ & $\mathrm{Y}$ & $\mathrm{Y}$ & $\mathrm{N}$ & $\mathrm{Y}$ & $\mathrm{Y}$ & $\mathrm{Y}$ & $\mathrm{Y}$ & $\mathrm{Y}$ & $\mathrm{N}$ & $\mathrm{Y}$ \\
\hline Taborri $(2015)^{30}$ & $\mathrm{Y}$ & $\mathrm{Y}$ & $\mathrm{Y}$ & $\mathrm{Y}$ & $\mathrm{Y}$ & $\mathrm{Y}$ & $\mathrm{Y}$ & $\mathrm{Y}$ & $\mathrm{Y}$ & $\mathrm{Y}$ & $\mathrm{Y}$ & $\mathrm{Y}$ & $\mathrm{N}$ & $\mathrm{Y}$ & $\mathrm{Y}$ & $\mathrm{Y}$ & $\mathrm{Y}$ & $\mathrm{N}$ & $\mathrm{N}$ & $\mathrm{Y}$ \\
\hline $\operatorname{Mazza}(2010)^{20}$ & $\mathrm{Y}$ & $\mathrm{Y}$ & $\mathrm{Y}$ & $\mathrm{Y}$ & $\mathrm{Y}$ & $\mathrm{Y}$ & $\mathrm{Y}$ & $\mathrm{Y}$ & $\mathrm{Y}$ & $\mathrm{Y}$ & $\mathrm{Y}$ & $\mathrm{Y}$ & $\mathrm{N}$ & $\mathrm{Y}$ & $\mathrm{Y}$ & $\mathrm{Y}$ & $\mathrm{Y}$ & $\mathrm{Y}$ & $\mathrm{N}$ & $\mathrm{Y}$ \\
\hline
\end{tabular}




\section{Introduction}

1 Were the aims/objectives of the study clear?

\section{Methods}

2 Was the study design appropriate for the stated aim(s)?

3 Was the sample size justified?

4 Was the target/reference population clearly defined? (Is

it clear who the research was about?)

5 Was the sample frame taken from an appropriate population base so that it closely represented the target/reference population under investigation?

6 Was the selection process likely to select subjects/participants that were representative of the target/reference population under investigation?

7 Were measures undertaken to address and categorise non-responders?

8 Were the risk factor and outcome variables measured appropriate to the aims of the study?

9 Were the risk factor and outcome variables measured correctly using instruments/ measurements that had been trialled, piloted or published previously?

10 Is it clear what was used to determined statistical significance and/or precision estimates? (eg, p values, CIs)

11 Were the methods (including statistical methods) sufficiently described to enable them to be repeated?

\section{Results}

12 Were the basic data adequately described?

13 Does the response rate raise concerns about non-response bias?

14 If appropriate, was information about non-responders described?

15 Were the results internally consistent?

16 Were the results for the analyses described in the methods, presented?

\section{Discussion}

17 Were the authors' discussions and conclusions justified by the results?

18 Were the limitations of the study discussed?

Other

19 Were there any funding sources or conflicts of interest that may affect the authors' interpretation of the results?

20 Was ethical approval or consent of participants attained?

\section{Search strategies (model) MEDLINE and PubMed}

1 infant

2 child

3 children

4 or/1-3

5 walk

6 walking

7 locomotion

8 gait

9 or/6-8

10 sensor

11 gyroscope

12 inertial

13 accelerometer

14 acceleration

15 or $/ 10-14$

\section{Acknowledgments}

This review was conducted during a scholarship supported by the International Cooperation Program CAPES/PROSUP at University Nove de Julho and was funded by CAPES - the Brazilian Federal Agency for the Support and Evaluation of Graduate Education within the Brazilian Ministry of Education.

\section{Corresponding author}

Paulo Roberto Fonseca Junior

Rua Padre João Gonçalves, 116 - Pinheiros - São Paulo/ SP Brazil - CEP: 05432-040

E-mail:paulofonseca28@gmail.com

Editor: Kátia D’Angelis. UNIFESP, São Paulo, SP, Brazil

Manuscript received on July 11, 2019

Manuscript accepted on May 4, 2020

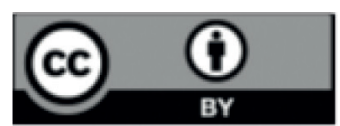

Motriz. The Journal of Physical Education. UNESP. Rio Claro, SP, Brazil - eISSN: 1980-6574 - under a license Creative Commons - Version 4.0 